Journal of Intelligent Systems. Volume 17, Issue Supplement, Pages 133-168.

DOI: 10.1515/JISYS.2008.17.S1.133, May 2011

\title{
Genetic Algorithm Optimization and Control System Design of Flexible Structures
}

\author{
M. O. Tokhi, M. Z. Md Zain, M. S. Alam, F. M. Aldebrez, \\ S. Z. Mohd Hashim and I. Z. Mat Darus \\ Department of Automatic Control and System Engineering, \\ University of Sheffield, UK
}

\begin{abstract}
This paper presents an investigation into the deployment of genetic algorithm (GA)based controller design and optimization for vibration suppression in flexible structures. The potential of GA is explored in three case studies. In the first case study, the potential of GA is demonstrated in the development and optimization of a hybrid learning control scheme for vibration control of flexible manipulators. In the second case study, an active control mechanism for vibration suppression of flexible beam structures using GA optimization technique is proposed. The third case study presents the development of an effective adaptive command shaping control scheme for vibration control of a twin rotor system, where GA is employed to optimize the amplitudes and time locations of the impulses in the proposed control algorithm. The effectiveness of the proposed control schemes is verified in both an experimental and a simulation environment, and their performances are assessed in both the time and frequency domains.
\end{abstract}

\section{KEYWORDS}

active vibration control, command shaping, flexible systems, genetic algorithm, hybrid learning control, optimization.

Reprint requests to: M.O. Tokhi, Department of Automatic Control and System Engineering, The University of Sheffield, UK; e-mail: o.tokhi@sheffield.ac.uk 


\section{INTRODUCTION}

Genetic algorithm (GA) is one of the global stochastic search algorithms based on natural biological evolution (Goldberg, 1989; Holland, 1975). GA is a parallel search technique that emulates the laws of evolution and genetics to yield optimal solutions to complex optimization problems. Initially, GA has been most widely and successfully applied to off-line design applications. In the field of control systems engineering, these applications include controller design and optimization, model identification, robust stability analysis, and fault diagnosis (Kristinsson \& Dumont, 1992; Luh \& Wu, 1999). In some instances, GA has been used as the sole means of design. In others, it has been combined with existing methods. For example, GA has been combined with other intelligent techniques and has incorporated a process of learning that can make appropriate, autonomous decisions.

In the early 1990s, GA was first investigated as an alternative means of tuning PID (Proportional-Integral-Derivative) controllers. Oliveira et al. (1991) used a standard GA to determine initial estimates for the values of PID parameters. They applied their methodology to a variety of classes of linear time-invariant (LTI) systems, encompassing minimum-phase, non-minimum phase, and unstable systems. Moreover, Onnen et al. (1995) applied GA to the determination of an optimal control sequence in model-based predictive control (MBPC). Particular attention was paid to MBPC for nonlinear systems with input constraints. Specialised genetic coding and operators were developed, with the aim of preventing the generation of infeasible solutions. The resulting scheme was applied to a simulated batch-fed fermenter, with favourable results reported (compared to the traditional branch-and-bound method) for long control horizons. A further approach to controller design using GA is to apply the methodology indirectly. In such a scheme, the GA manipulates input parameters to an established controller design process, which in turn produces the final controller. The linear quadratic Gaussian (LQG) method and the H-infinity control scheme have both been utilised in this manner (Chen \& Cheng, 1998; Dakev et al., 1997; Itoh et al., 2004; Mei \& Goodall, 2000; Whidborne et al., 1994). In this investigation, randomly selected parameters are optimized in different cases by applying the working mechanism of GA.

The limitations of conventional controllers for application to complicated, dynamic systems have motivated research into the so-called intelligent control 
systems. GA has been used in attempts to optimize various aspects of intelligent controllers (Linkens \& Nyongesa, 1996). In fuzzy control, GA has been used to generate the fuzzy rule base, and to tune the associated membership function parameters (Linkens \& Nyongesa, 1995; Varsek et al., 1993). In neural control, GA can function as an alternative choice to learning the weight values (Ichikawa and Sawa, 1992). GA has also been shown to be capable of optimising the topology of a neural network (Leung et al., 2003).

GA has also been used in robotics for both path planning and design of behavioral controllers. Rana and Zalzala (1997) applied GA to the collision-free path planning of robot arms. Each chromosome consisted of a floating-point vector representation of via points (between each end of the path). The actual path was then computed by fitting cubic splines to the points. The cost function was a weighted sum of the path length, the number of collisions, and the distribution of via points.

This paper aims to demonstrate the potential of GA in both control design and optimization for tracking control and vibration suppressions in flexible maneuvering systems. Flexible maneuvering systems exhibit many advantages over their rigid counterparts. For instance, they have higher manipulation speed, are more maneuverable and transportable, and are safer to operate because of their lighter weight, lower power consumption, smaller actuators requirement, and reduced inertia. However, the control task of such systems is a challenging problem, due to nonlinearities and flexible dynamics that lead to vibration during operation (Mohamed \& Tokhi, 2002). Moreover, obtaining accurate model of such systems is a nontrivial task due to the non-minimum phase characteristics of the system. The control strategies employed for such complex systems are classified as feedforward, feedback, and combined feedback feedforward control schemes. Three control schemes are designed and implemented on three different flexible rigs in this study. In the first case study, GA is used to develop and optimize a hybrid learning control scheme for vibration control of a flexible manipulator. In the second case study, an active control mechanism for vibration suppression of flexible beam structures using GA optimization is proposed. In the third case study, GA is employed to optimize the amplitudes and time locations of an adaptive command shaping control scheme for vibration control of a twin rotor system. The effectiveness of the proposed control schemes is verified in experimental and simulation environments. The performances of the control schemes are assessed in both the time and frequency domains. 
From an operational perspective, a GA comprises two basic elements of individuals, i.e., potential solutions (the population) and a set of biologically inspired

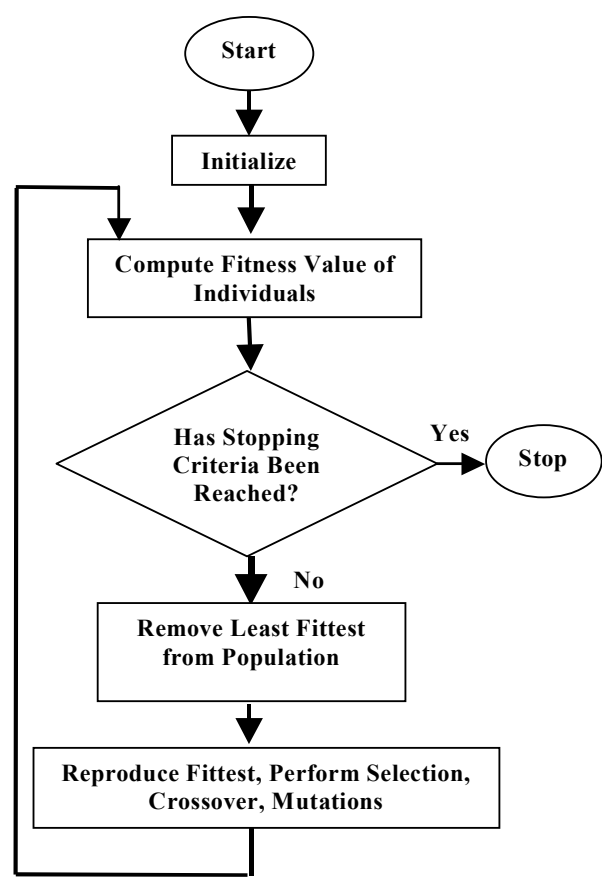

Fig. 1: Flowchart of basic GA

operators active over the population. A new set of approximations/ solutions is created at each generation, by the process of selecting individuals according to their level of fitness in the problem domain and breeding them together using the operators. This process leads to the evolution of populations of individuals that are better suited to their environment than the individuals that they were created from, just as in natural adaptation (Goldberg, 1989; Holland, 1975). The search process of a GA is carried out according to the flowchart depicted in Figure 1:

To begin the search cycle, a fitness or performance measure must be defined as a function of members of the population. Secondly, several parameters must be set. These include as a minimum: the population size, and frequency of mutation and crossover. An initial population is then generated (randomly, possibly with constraint), and the fitness of each individual is computed. If the fitness of any 
individual satisfies the stopping criteria, the search is terminated and the solution returned. Otherwise, a new generation is created through reproduction, crossover, mutation, and possibly other operations.

\section{HYBRID LEARNING CONTROL OF FLEXIBLE MANIPULATOR SYSTEMS}

Many industrial applications of robot manipulators involve iterative repeated cycles of events. Thus, it is important to minimize errors in trajectory tracking of such manipulators, and this can be achieved with suitable learning strategies. The basic idea behind iterative learning control (ILC) is that the controller should learn from previous cycles and perform better during every subsequent cycle. Such ideas were presented by Arimoto et al. in 1984 who proposed a learning control scheme called the improvement process, and since then many papers have addressed robot control in combination with iterative learning control, (Craig, 1988; Horowitz, 1993; Panzieri \& Ulivi, 1995).

In this work, ILC is studied as a complement to conventional feedforward and feedback control scheme for tracking control and vibration reduction of a constrained planar single-link flexible manipulator. A simulation environment is developed within Simulink and Matlab for evaluation of performance of the control strategies. The dynamic model of the flexible manipulator is derived using the finite element (FE) method. Previous simulation and experimental studies have shown that the FE method gives an acceptable dynamic characterization of the actual system (Tokhi et al., 1997). Moreover, a single element is sufficient to describe the dynamic behavior of the manipulator reasonably well. To demonstrate the effectiveness of the proposed control schemes, initially a joint-based collocated (JBC) PD controller utilizing hub-angle and hub-velocity feedback is developed for control of rigid body motion. This is then extended to incorporate an ILC scheme for vibration suppression of the manipulator. Simulation results of the response of the manipulator with the controller are presented in time and frequency domains. The performance of the hybrid learning control scheme is assessed in terms of input tracking and level of vibration reduction in comparison to the response with genetic algorithm optimized parameters.

\subsection{The Flexible Manipulator System}

Figure 2 shows a laboratory-scale single-link experimental rig used in this work. The manipulator can be considered as a pinned-free flexible arm, which can bend 
freely in the horizontal plane but is relatively stiff in vertical bending and torsion (Tokhi \& Azad, 1997). The manipulator is an aluminum type with dimensions $900 \times$ $19.008 \times 3.2004 \mathrm{~mm}^{3}, E=71 \times 10^{9} \mathrm{~N} / \mathrm{m}^{2}, I=5.253 \times 10^{-11} \mathrm{~m}^{4}$, and $I_{k}=5.8298 \times 10^{-4} \mathrm{kgm}^{2}$.
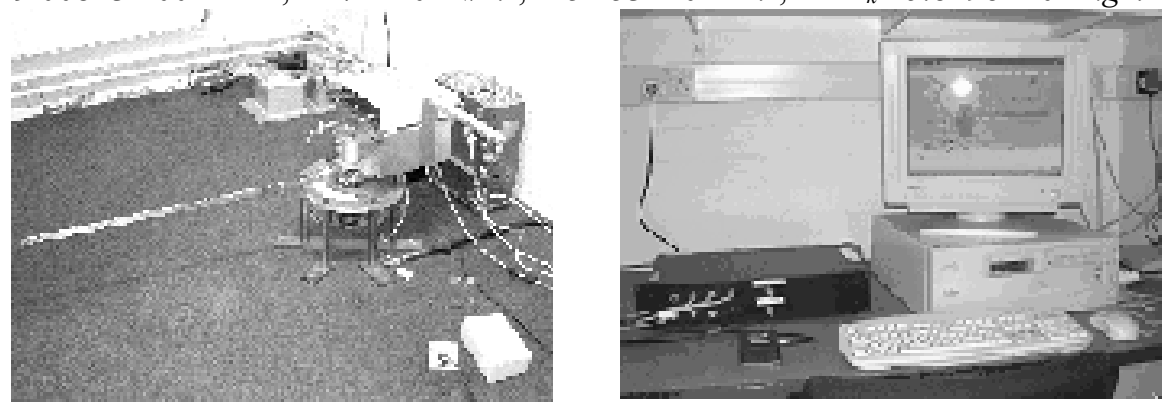

Fig. 2: The laboratory-scale single-link flexible manipulator

The digital processor used is an IBM compatible PC based on an Intel(r) Celeron ${ }^{\mathrm{TM}}$ processor. Data acquisition and control are accomplished through the utilization of PCL-812PG board. This board can provide a direct interface between the processor, actuator and sensors. A simulation algorithm characterizing the dynamic behavior of the manipulator has previously been developed using the finite element (FE) method (Tokhi et al., 1997, 1999). This is used in this work as a platform for theoretical test and evaluation of the proposed control approaches.

\subsection{Collocated PD Control}

A common strategy in the control of manipulator systems involves the utilization of PD feedback of collocated sensor signals. Such a strategy is adopted at this stage of the investigation here. A block diagram of the PD controller is shown in Figure 3, where $K_{p}$ and $K_{v}$ are the proportional and derivative gains respectively $\theta, \dot{\theta}$ and $\alpha$ represent hub angle, hub velocity and end-point residual respectively, $R_{f}$ is the reference hub angle and $A_{c}$ is the gain of the motor amplifier. Here the motor/amplifier set is considered as a linear gain, $A_{c}$, as the set is found to function linearly in the frequency range of interest. To design the PD controller a linear statespace model of the flexible manipulator was obtained by linearizing the equations of motion of the system. The first two flexible modes of the manipulator were assumed to be dominantly significant. Further details of derivation of the equation of the flexible 
manipulator closed-loop characteristic can be found in (Tokhi and Azad, 1996). In this study, the root locus approach is utilized to design the PD controller. Analyses of the root locus plot of the system show that dominant poles with maximum negative real

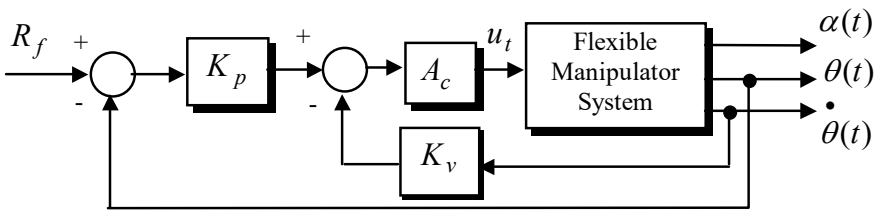

Fig. 3: The collocated PD control structure

parts could be achieved with $Z=K_{p} / K_{v} \approx 2$ and by setting $K_{p}$ between 0 and 1.2 (Tokhi \& Azad, 1996).

\subsection{Hybrid Collocated PD with Iterative Learning Control}

A hybrid collocated PD control structure for control of rigid-body motion of the flexible manipulator with ILC is proposed in this section. In this study, an ILC scheme is developed using PD-type learning algorithm. Iterative learning control has been an active research area for more than a decade, mainly inspired by the pioneering work of Arimoto et al. (1984). Learning control begun with the fundamental principle that repeated practice is a common mode of human learning. Given a goal (regulation, tracking, or optimization), learning control, or more specifically, iterative learning control refers to the mechanism by which necessary control can be synthesized by repeated trials. A typical learning algorithm is given as:

$$
\hbar_{k+1}=\hbar_{k}+\Phi e_{k}+\Gamma \dot{e}_{k}
$$

where

$\hbar_{k+1}$ is the next control signal

$\hbar_{k} \quad$ is the current control signal

$e_{k} \quad$ is the current positional error input, $e_{k}=\left(x_{d}-x_{k}\right) \Phi, \Gamma$ are suitable positive definite constants (or learning parameters)

Obviously, the algorithm contains a constant and derivative coefficient of the error. In other words, the expression can be simply called proportional-derivative or 
PD type learning algorithm. A slightly modified learning algorithm to suit the application is employed here. Instead of using the absolute position tracking error $e_{k}$, a sum-squared tracking error is used.

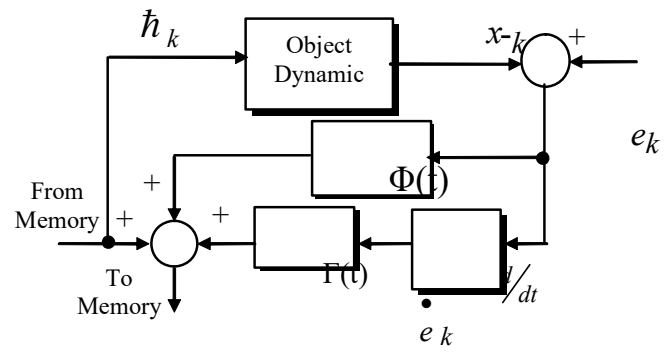

Fig. 4: PD type learning algorithm

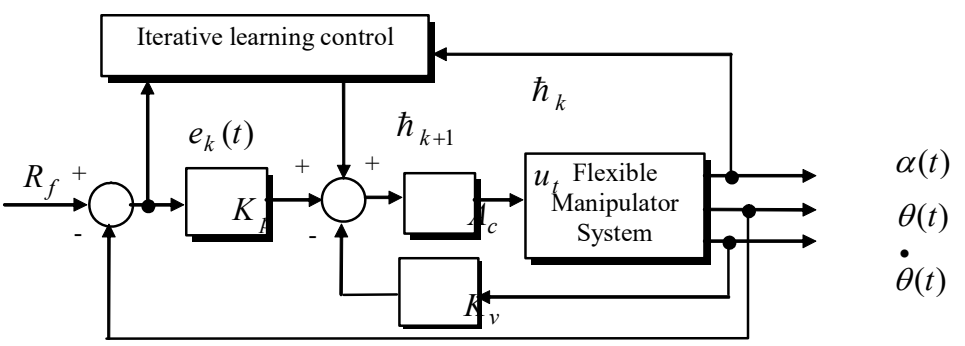

Fig. 5: The collocated PD with iterative learning control structure

Figure 4 shows a block diagram describing the above expression. This is used with PD collocated control, to realise the hybrid collocated PD with ILC. This is shown in Figure 5.

\subsection{GA Based Hybrid Learning Control}

The performance of a PD-type learning control depends upon the proportional gain $\Phi$ and derivative gain $\Gamma$. The stability, settling time, maximum overshoot and many other system performance indicators depend upon the values of $\Phi$ and $\Gamma$. The proposed strategy utilizes GA as an optimization and search tool to determine the optimum values of the gains. The performance index or the cost function chosen is the error in the system output to reach and stay within a range specified by 
absolute percentage of the final value. Hence, the role of GA is to find optimum vales of gains $\Phi$ and $\Gamma$. In this case, integral of squared error is used as the criterion for minimising the error:

$$
I A E=\int_{0}^{T}\left|\frac{\sum \text { Error }^{2}}{N}\right| d t
$$

Error $=r(t)-y(t), \mathrm{N}=$ number of samples;

where, $r(t)=$ reference input $y(t)=$ measured variable

The above error criterion is used with GA based tuning. The GA initializes a random set of population of the two variables. The algorithm evaluates all members of the population based on the specified performance index. The algorithm then applies GA operators such as reproduction, crossover and mutation to generate a new set of population based on the performance of members of the population (Goldberg, 1989). The best member or gene of the population is chosen and saved for next generation. It again applies the operators and selects the best gene among the new population. The best gene of the new population is compared to best gene of previous population. If a predefined termination criterion is not met, again a new population is obtained as above. The termination criterion may be formulated as the magnitude of difference between index value of previous generation and present generation becoming less than a pre-specified value. The process continues till the termination criterion is fulfilled.

\subsection{Results and Discussion}

In this section, the proposed control schemes are implemented and tested within the simulation and experimental environments of the flexible manipulator and the corresponding results are presented. The manipulator is required to follow a trajectory at $\pm 75^{\circ}$ as shown in Figure 6. System responses, namely the hub-angle, hub-velocity and end-point acceleration are observed. To investigate the vibration of the system in the frequency domain, power spectral density (SD) of response at the end-point is obtained.

In the collocated control scheme, the design of PD controller was based on root locus analysis, from which $K_{p} K_{v}$ and $A_{c}$ were deduced as $0.64,0.32$, and 0.01 respectively. The closed-loop parameters with the PD control are then used to 
F.M. Aldebrez, et al.

design and evaluate the performance of iterative learning control schemes in terms of input tracking capability and level of vibration reduction. The (PD-ILC) scheme was

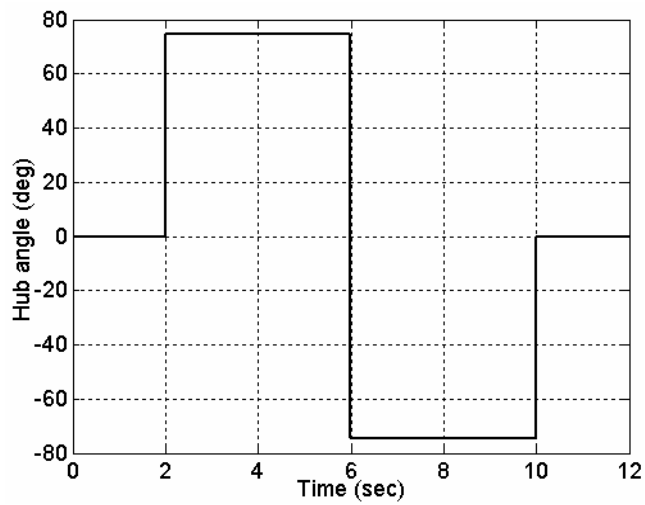

Fig. 6: The reference hub angle

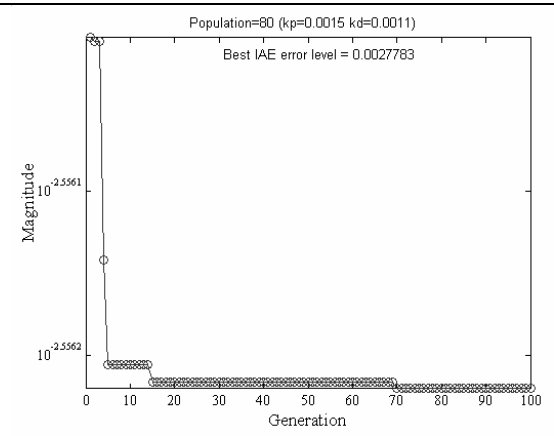

Fig. 7: Objective value vs number of Fig. 8: Objective value vs number of generations (shimulated)

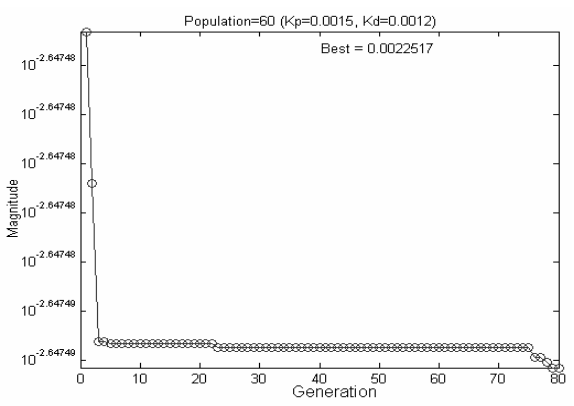

generations (Experimental)

designed based on the dynamic behavior of the closed-loop system. In this paper the parameters of the learning algorithm $\Phi$ and $\Gamma$ were tuned using the GA. With GA optimization, the parameters were deduced as 0.0015 and 0.0011 in simulation and 0.0015 and 0.0012 with the experimental system respectively. The GA was designed with 80 individuals for simulation and 60 for the experimental system in each generation. The maximum number of generations was set to 100 for simulation and 80 for the experimental system. Figures 7 and 8 and Table 1 show the algorithm 
convergence as a function of generations and the parameter values used in the GA algorithm respectively.

TABLE 1

Algorithm parameters for PD-type learning

\begin{tabular}{|c|c|}
\hline Parameter & Setting \\
\hline Generation gap & 0.9 \\
\hline Precision & 14 \\
\hline Crossover rate & 0.8 \\
\hline Mutation rate & 0.025 \\
\hline
\end{tabular}

Figure 9 shows the corresponding responses of the GA optimization process with HLC. It is noted that in this case the GA based hybrid learning scheme performs much better than the collocated PD control. GA based hybrid learning optimizes the proportional and derivative gains of the controller such that the settling time of the system is minimized. This is also expected to reduce the settling time of the response.

\section{ACTIVE VIBRATION CONTROL OF A FLEXIBLE BEAM SYSTEM}

Active vibration control (AVC) consists of artificially generating cancelling sources to destructively interfere with the unwanted source and thus result in reduction in the level of the disturbance (vibration) at desired locations. This is realised by detecting and processing the vibration by a suitable electronic controller so that when superimposed on the disturbance, cancellation occurs (Tokhi, 1997; Tokhi \& Leitch, 1992; Tokhi \& Veres, 2002). In this study, an investigation is carried out into the development of an adaptive active control mechanism for vibration suppression of flexible structures using GA.

Due to the broadband nature of the disturbances, it is required that the control mechanism in an AVC system realises suitable frequency-dependent characteristics so that cancellation over a broad range of frequencies is achieved. In practice, the spectral contents of the disturbance as well as the characteristics of system components are, in general, subject to variation, giving rise to time-varying 
F.M. Aldebrez, et al.

phenomena. This implies that the control mechanism is further required to be intelligent enough to track these variations so that the desired level of performance is achieved and maintained (Tokhi \& Veres, 2002).

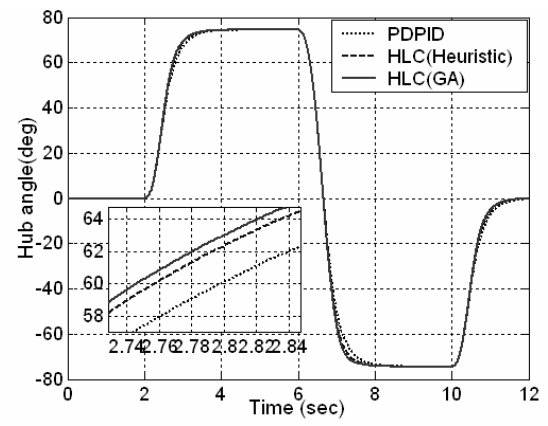

Simulated

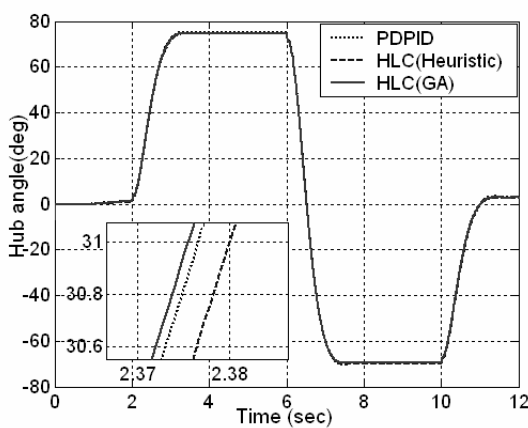

Experimental

(a) Hub angle

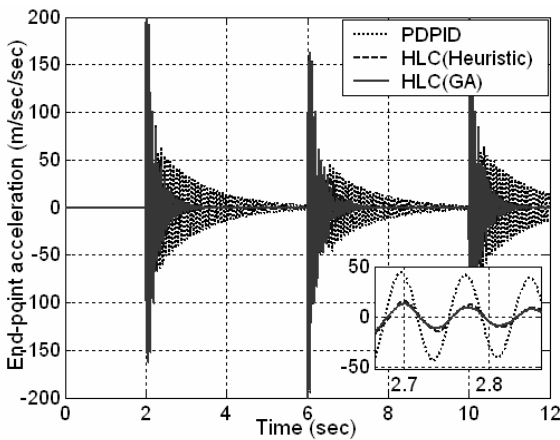

Simulated

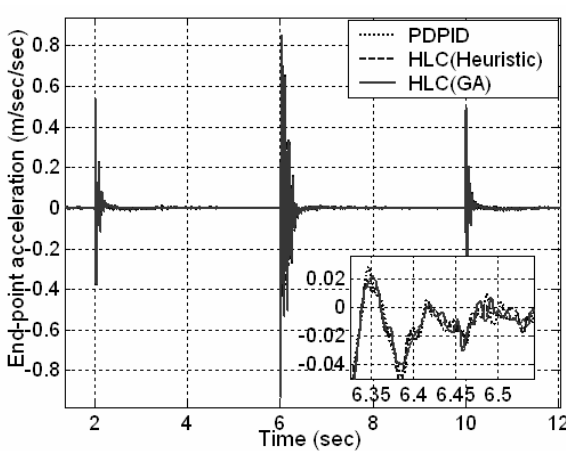

Experimental

(b) End-point acceleration (Time domain)

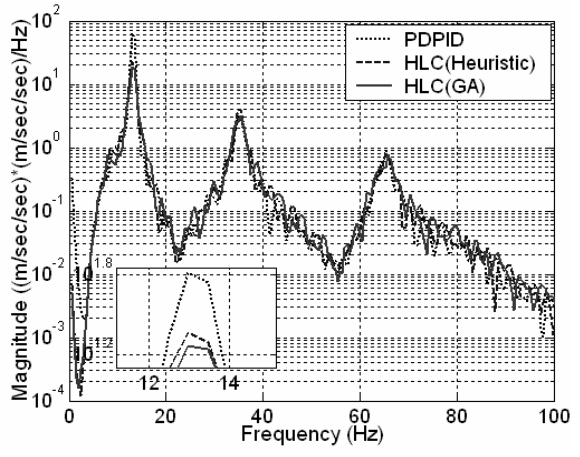

Simulated

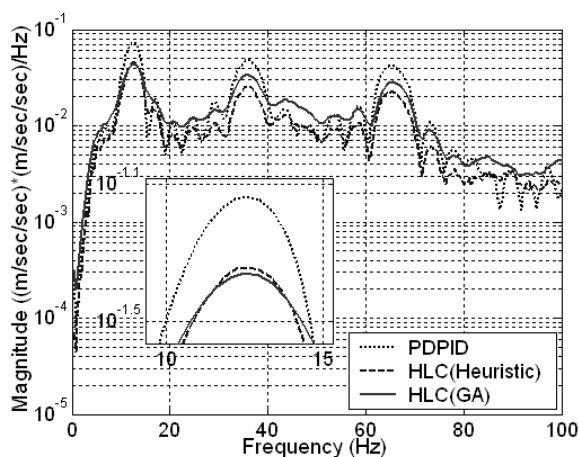

Experimental 
(c) End-point acceleration (Spectral density)

Fig. 9: Response of the simulated and experimental manipulator system without payload

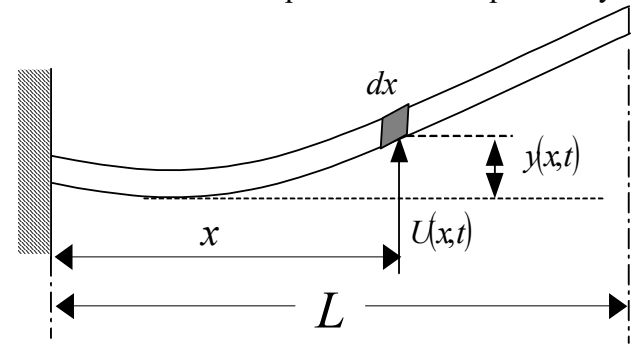

Fig. 10: Fixed-free beam

A flexible beam in fixed-free mode, a representation of aircraft wing or robot arm, is considered in this study. Such a system has an infinite number of modes although in most cases the lower modes are the dominant ones requiring attention. The unwanted vibrations in the structure are assumed to be due to a single point disturbance of broadband nature. First order central finite difference (FD) methods are used to study the behavior of the beam and develop a suitable test and verification platform. An AVC system is designed using a single-input single-output (SISO) control structure to yield optimum cancellation of broadband vibration at an observation point along the beam. Here, GA uses a direct analogy of natural evolution. Through the genetic evolution method, an optimal solution can be found and represented by the final winner of the genetic game. The development of an active control mechanism for vibration suppression of flexible beam structures using GA optimization technique is proposed and its performance is assessed and discussed.

\subsection{The Flexible Beam System}

Figure 10 shows a flexible beam in fixed-free mode where $U(x, t)$ represents an applied force at a distance $x$ from the fixed end at time $t$ and $y(x, t)$ is the resulting beam deflection from its stationary position at the point where the force has been applied. $L$ is the length of the beam and $d x$ is a differential length of the beam.

The motion of a beam in transverse vibration in response to an applied force $U(x, t)$ is governed by the well-known fourth-order partial differential equation (PDE) (Hossain, 1996; Kourmoulis, 1990). 


$$
\mu^{2} \frac{\partial^{4} y(x, t)}{\partial x^{4}}+\frac{\partial^{2} y(x, t)}{\partial t^{2}}=\frac{1}{m} U(x, t)
$$

where $\mu$ is a beam constant given by $\mu^{2}=\frac{\mathrm{EI}}{\rho \mathrm{A}}$ with $E, I, \rho$ and $A$ representing Young

modulus, moment of inertia of the beam, mass density and cross-sectional area respectively, and $m$ is the mass of the beam. The corresponding boundary conditions at the fixed and free ends of the beam are given as:

$$
\begin{array}{r}
y(0, t)=0 \text { and } \frac{\partial y(0, t)}{\partial x}=0 \\
\frac{\partial^{2} y(L, t)}{\partial x^{2}} \text { and } \frac{\partial^{3} y(L, t)}{\partial x^{3}}=0
\end{array}
$$

Note that the model in Eq. (3) does not incorporate damping. The FD method is used as a numerical solution to the PDE in Eq. (3) (Hossain, 1996). This involves a discretization of the beam into a finite number of equal length sections, each of length $\Delta x$, and the beam motion (deflection) for the end of each section is considered at equally spaced time steps of duration $\Delta t$. Thus, denoting $y(x, t)$ to represent the beam deflection $y_{i, j}$ at point $i$ at time step $j$ and $y(x+v \Delta x, t+w \Delta t)$ by $y_{i+v, j+w}$ where $v$ and $w$ are non-negative integer numbers, the required relations for the simulation algorithm, characterising the behavior of the system can be approximated. Using first-order central FD methods based on Eqs. (3) to (5) give:

$$
\begin{aligned}
& y_{j+1}=-y_{j-1}-\lambda^{2} \mathbf{S} y_{j}+\frac{(\Delta t)^{2}}{m} U(x, t) \\
& y_{0, j}=0, \\
& y_{-1, j}=y_{1, j}, \\
& y_{n+1, j}=2 y_{n, j}-y_{n-1, j},
\end{aligned}
$$




$$
y_{n+2, j}=2 y_{n+1, j}-2 y_{n-1, j}+y_{n-2, j}
$$

where $\left.Y_{j}=\left[y_{1, j}\right], y_{2, j} \ldots y_{n, j}\right]^{T}$ and $\mathbf{S}$ is a matrix dependent on system parameters, number of segments $n$ and boundary conditions of the beam, and $\lambda_{2}=\frac{\left(\Delta t^{2}\right)}{\left(\Delta x^{4}\right)} \cdot \mu^{2}$

The stability of the algorithm in Eq. (6) is satisfied by $0<\lambda^{2} \leq 0.25$ (Kourmoulis, 1990). Considering an aluminium beam of length as $0.635, \mu$ as 1.351108 , mass as $0.0478 \mathrm{~kg}$ and $\lambda$ as 0.2948 , the first five resonance modes of the beam, as obtained through simulation exercise and verified by theoretical analysis, are located at $1.875 \mathrm{~Hz}, 11.751 \mathrm{~Hz}, 32.902 \mathrm{~Hz}, 64.476 \mathrm{~Hz}$ and $106.583 \mathrm{~Hz}$ respectively with the first two modes being the dominant ones.

\subsection{Direct-GA Active Vibration Control}

Figure 11 shows a schematic diagram of a SISO-AVC system. An unwanted (primary disturbance) point source emits broadband disturbance into the structure, which is detected by a detector, processed by a controller of suitable transfer characteristics and fed to a cancelling (secondary) point actuator. The secondary (control) signal thus generated, interferes with the disturbances to reduce the level of vibration at an observation point along the structure.

In this work, the aim of the controller design is to minimize the deflection $Y_{O}$ via $U_{C}$ for generating anti-phase control signal to counteract the vibration produced by $U_{D}$. Optimal vibration reduction can be achieved by feeding the observed signal $Y_{O}$ to the controller $C(z)$ via GA optimization routines (Hossain et al., 1995; Hossain and Tokhi, 1997). The fitness function in Eq. (5) is adopted in identifying the parameters of the controller transfer function. 


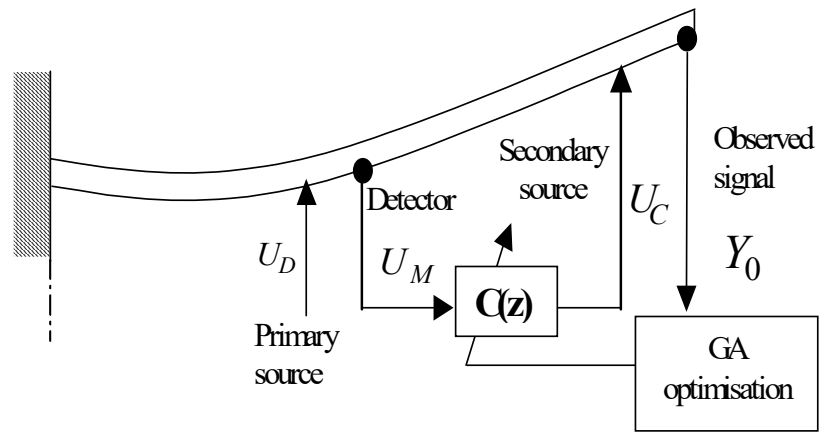

Fig. 11: Active vibration control (feedforward) structure

\subsection{Implementation and Results}

To study the performance of the GA-optimized controller, an aluminium type fixed-free beam with specifications given in section 3.1 was simulated. The beam was divided into 20 sections and a sampling time of $0.2 \mathrm{~ms}$ that satisfies the stability requirements of the FD simulation algorithm and is sufficient to cover all the resonance modes of vibration of the beam was used.

In order to allow nonlinear dynamics of the system be incorporated within the design, a uniformly random signal covering the dynamic range of interest of the system was used in the GA optimization process. The primary force was applied at grid point 13 and the secondary (control) signal was applied at grid point 15 . The detector and observer were placed at grid points 12 and 19 respectively.

Investigations were carried out using the GA with one step ahead (OSA) prediction and different initial values and operator rates. Satisfactory results were achieved with the following set of parameters:

- Generation gap:0.9

- Crossover rate:0.7

- Mutation rate:0.1

The GA controller model was observed with different orders and the best result was achieved with an order 6 . The GA was designed with 100 individuals in each generation. The maximum number of generations was set to 60 . The algorithm achieved the best mean-square error level of $2.238 \mathrm{e}-10$ in the $50^{\text {th }}$ generation. Figure 12 shows the algorithm convergence as a function of generations. Figure 13 shows 
the corresponding result of system performance with the GA optimization using random excitation as primary signal. As noted $21.718 \mathrm{~dB}, 6.990 \mathrm{~dB}, 17.074 \mathrm{~dB}$, $2.599 \mathrm{~dB}$ and $6.1470 \mathrm{~dB}$ vibration reduction was achieved at the first, second, third, fourth and fifth modes, respectively. To assess the performance of the GA controller further, pseudo random binary sequence (PRBS) excitation was used as the primary signal. The corresponding results are shown in Figure 14. It is noted that the spectral attenuation achieved at the resonance modes were $26.339 \mathrm{~dB}, 1.500 \mathrm{~dB}, 10.340 \mathrm{~dB}$ and $3.748 \mathrm{~dB}$ at the first, second, third and fifth respectively and reinforcement of $5.00 \mathrm{~dB}$ occurred at the fourth mode. It is therefore noted that the controller performs well with different input signals.

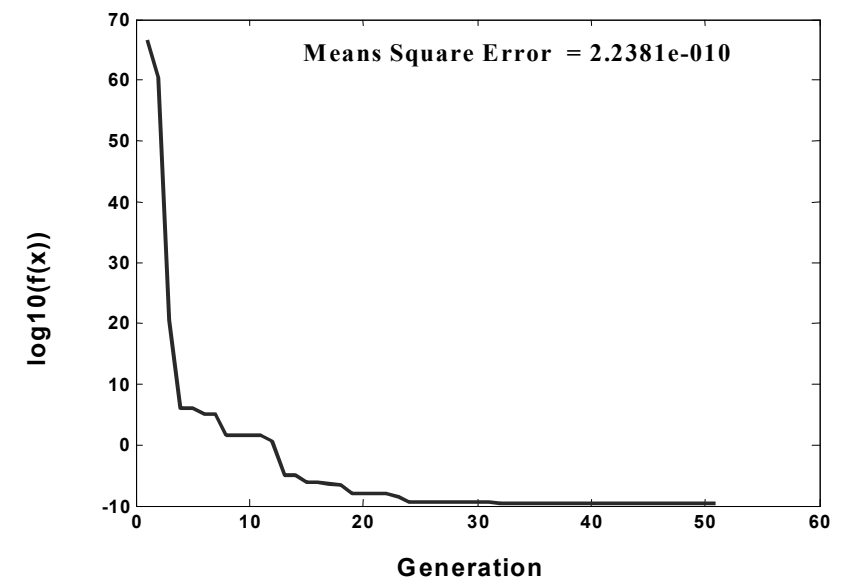

Fig. 12: Objective value vs number of generations

\section{ADAPTIVE COMMAND SHAPING FOR VIBRATION CONTROL OF A TWIN ROTOR SYSTEM}

The control of flexible maneuvering systems is a challenging problem due to the complex flexible dynamics and system nonlinearties. The control strategies employed for such complex systems are classified as feedforward, feedback and a 
F.M. Aldebrez, et al.

combination of these control schemes. Feedforward methods have been considered in vibration control for flexible systems where the control signal is developed by considering the physical and vibrational properties of the flexible system (Singer and Seering, 1990; Singhose et al., 1995). In this work, input-shaper with a sequence of three impulses is introduced as a feedforward technique for vibration control in the vertical movement of a twin rotor multi-input multi-output system (TRMS).

This study looks into the development of an effective adaptive commandshaping technique using GA for vibration control of a flexible system. An evolutionary method is introduced to adapt a command shaper used for vibration control of the vertical movement of a TRMS.

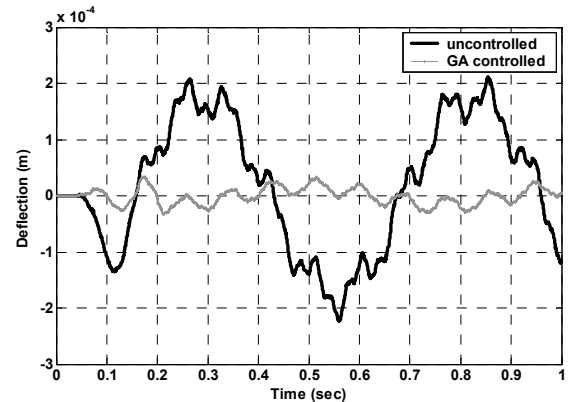

(a) System response at observation point in the time domain

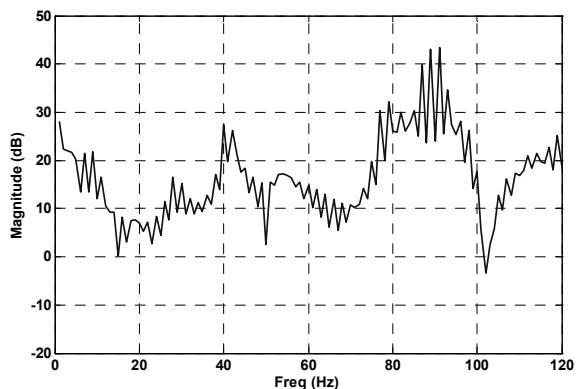

(c) Spectral attenuation

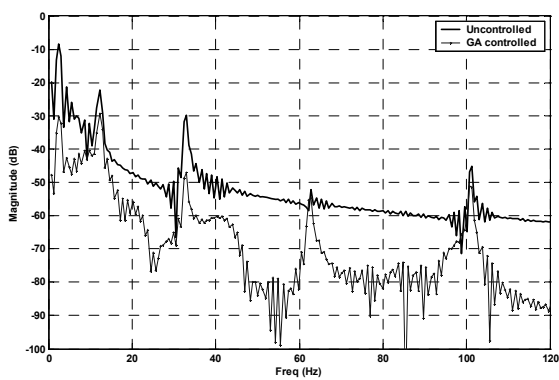

(b) System response at observation point in the frequency domain

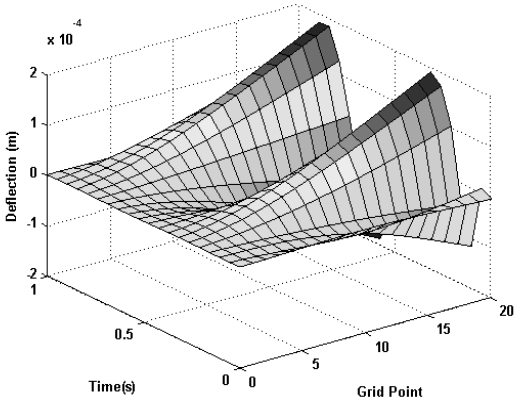

(d) System response in 3D before cancellation 


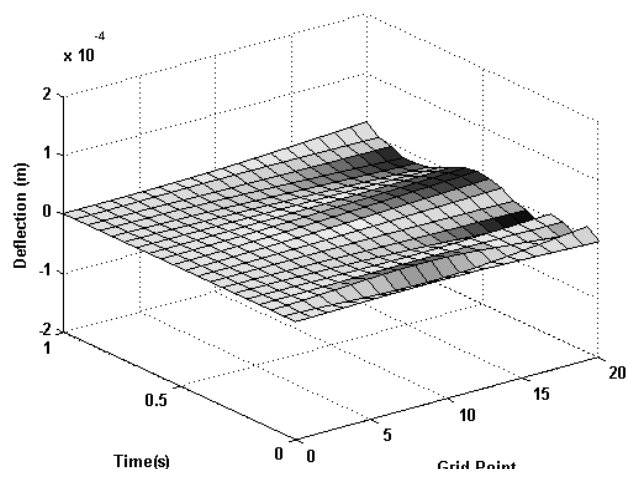

e) System response in 3D after cancellation

Fig. 13: Performance of GA-AVC using random excitation

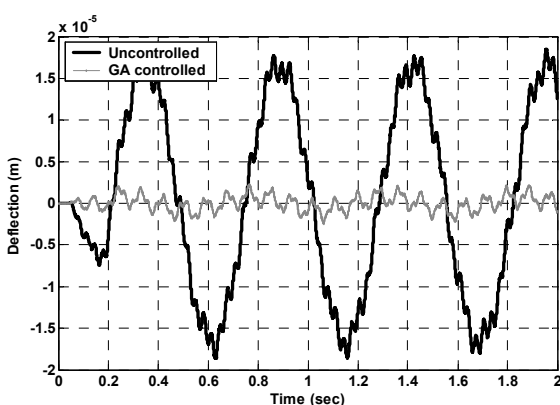

(a) System response at observation point in the time domain

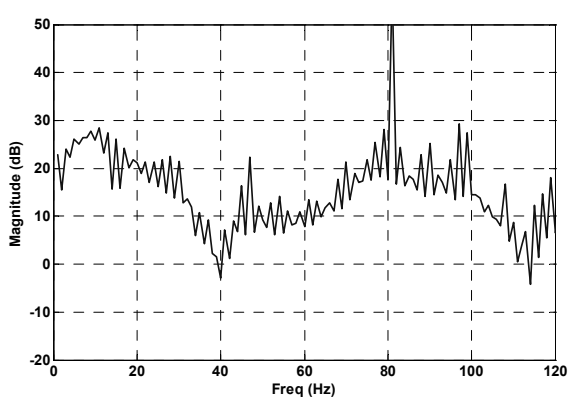

(c) Spectral attenuation

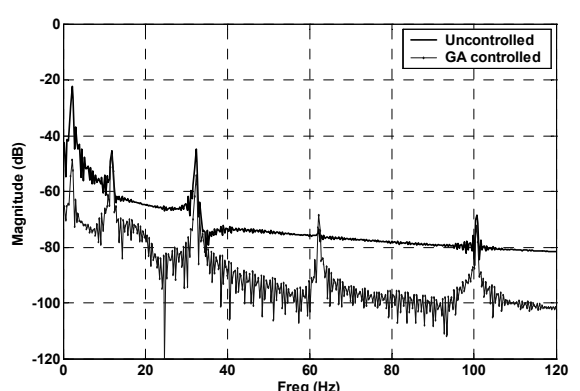

(b) System response at observation point in the frequency domain

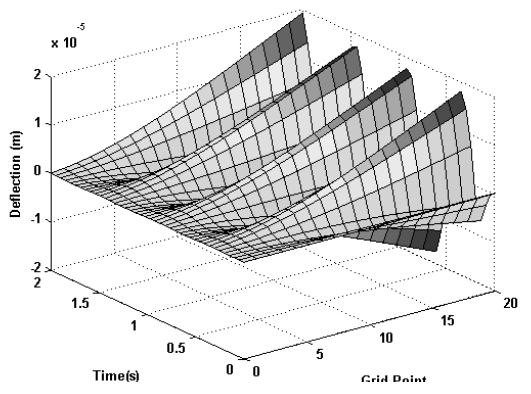

(d) System response in $3 \mathrm{D}$ before cancellation 


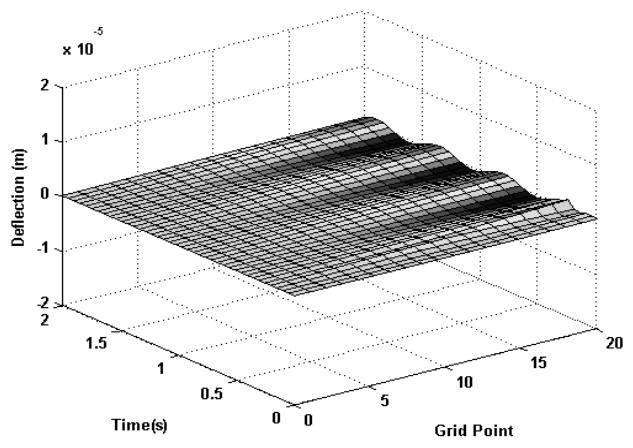

e) System response in 3D after cancellation

Fig. 14: Performance of GA-AVC using PRBS excitation

Command or input shaping, depicted in Figure 15, operates by taking the reference command and changing its shape so that it will not excite the flexible system modes and as a result the vibrations that would be associated with motion

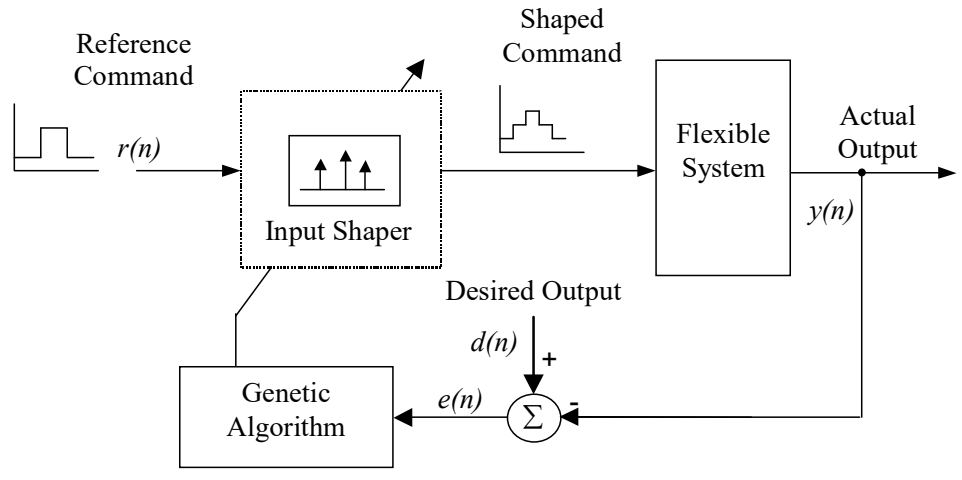

Fig. 15: Adaptive command shaping for vibration control

will be reduced. Since its introduction (Singer and Seering, 1990; Singhose et al., 1995), command shaping has been applied to the control of different types of flexible systems (Aldebrez et al., 2003; Mohamed and Tokhi, 2004). A properly designed command shaper cancels the resonance poles of the system regardless of 
given reference input to the system. However, designing an effective command shaper requires a priori knowledge of the system parameters. Recently, some efforts have been made to make the command shaper less sensitive to the uncertainty in system parameters and to make the command shaper adapt to the unknown system parameters (Bodson, 1998; Sungsoo, 1999). This research is an effort to develop an effective adaptive command-shaping control scheme using GA in time domain. Genetic algorithm is one of the global stochastic search algorithms based on natural biological evolution (Goldberg, 1989; Holland, 1975). The potential of GA is investigated to optimize the amplitudes and time-location of the impulses in the proposed input shaper illustrated in Figure 15.

\subsection{The TRMS Setup}

The TRMS, shown in Figure 16, is a laboratory set-up designed for control experiments (Feedback Instruments Ltd., 1996). In certain aspects it behaves like a helicopter. The TRMS rig consists of a beam pivoted on its base in such a way that it

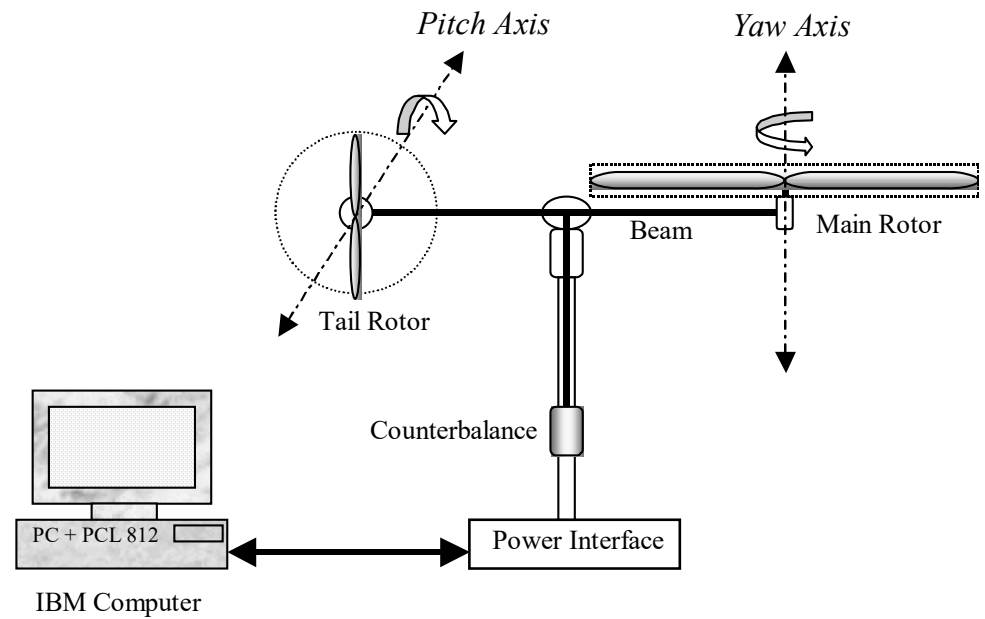

Fig. 16: The schematic diagram of the TRMS

can rotate freely both in the horizontal and vertical directions producing yaw and 
pitch movements, respectively. At both ends of the beam there are two rotors driven by two d.c. motors. The main rotor produces a lifting force allowing the beam to rise vertically making a rotation around the pitch axis (vertical angle). While, the tail rotor (smaller than the main rotor) is used to make the beam turn left or right around the yaw axis (horizontal angle). In a typical helicopter, the aerodynamic force is controlled by changing the angle of attack of the blades. The laboratory set-up is constructed so that the angle of attack of the blades is fixed and the aerodynamic force is controlled by varying the speed of the motors. Therefore, the control inputs are supply voltages of the d.c. motors. A change in the voltage value results in a change in the rotational speed of the propeller, which results in a change in the corresponding position of the beam (Feedback Instruments Ltd., 1996).

\subsection{Feedforward Vibration Control}

The input shaping method involves convolving a desired command with a sequence of impulses (Singer \& Seering, 1990). The design objectives are to determine the amplitude and time location of the impulses. A vibratory system can be modelled as a superposition of second order systems each with a transfer function:

$$
G(s)=\frac{\omega_{n}^{2}}{s^{2}+2 \zeta \omega_{n} s+\omega_{n}^{2}}
$$

where, $\omega_{n}$ is the natural frequency and $\zeta$ is the damping ratio of the system. Thus, the impulse response of the system at time $t$ is:

$$
y(t)=\frac{A \omega_{n}}{\sqrt{1-\zeta^{2}}} e^{-\zeta \omega_{n}\left(t-t_{0}\right)} \sin \left[\omega_{n} \sqrt{1-\zeta^{2}}\left(t-t_{0}\right)\right]
$$

where, $A$ and $t_{0}$ are the amplitude and time-location of the impulse, respectively. Furthermore, the response to a sequence of impulses can be obtained using the superposition principle. Thus, for $N$ impulses, with $\omega_{d}=\omega_{n} \sqrt{1-\zeta^{2}}$, the impulse response can be expressed as:

$y(t)=M \sin \left(w_{d} t+\alpha\right)$

where, 


$$
\begin{aligned}
& M=\sqrt{\left(\sum_{i=1}^{N} B_{i} \cos \phi_{i}\right)^{2}+\left(\sum_{i=1}^{N} B_{i} \sin \phi_{i}\right)^{2}} \\
& B_{i}=\frac{A \omega_{n}}{\sqrt{1-\zeta^{2}}} e^{-\zeta \omega_{n}\left(t_{N}-t_{i}\right)} \\
& \phi_{i}=\omega_{d} t_{i} \rightarrow \quad \alpha=\tan ^{-1}\left(\sum_{i=1}^{N} \frac{B_{i} \cos \phi_{i}}{B_{i} \sin \phi_{i}}\right)
\end{aligned}
$$

and $A_{i}$ and $t_{i}$ are the magnitudes of the impulses and their time-location, respectively. The residual single-mode vibration amplitude of the impulse response is obtained at the time of the last impulse, $t_{N}$, as:

$$
V=\sqrt{V_{1}^{2}+V_{2}^{2}}
$$

where.

$$
V_{2}=\sum_{i=1}^{N} \frac{A_{i} \omega_{n}}{\sqrt{1-\zeta^{2}}} e^{-\zeta \omega_{n}\left(t_{N}-t_{i}\right)} \sin \left(\omega_{d} t_{i}\right)
$$

To achieve zero vibration after the last impulse, it is required that both $V_{l}$ and $V_{2}$ in Eq. (10) are independently zero. Furthermore, to ensure that the shaped command input produces the same rigid-body motion as the unshaped command, it is required that the sum of amplitudes of the impulses is unity (Singer and Seering, 1990). To avoid response delay, the first impulse is selected at time $t_{l}=0$. Hence, setting $V_{l}$ and

$V_{2}$ in Eq. (10) to zero, $\sum_{i=1}^{N} A_{i}=1$ and solving for the second derivative of the vibration in Eq. (10), will produce a four-impulse sequence with set of parameters given by:

$$
t_{1}=0 \quad t_{2}=\frac{\pi}{\omega_{d}} \quad t_{3}=\frac{2 \pi}{\omega_{d}} \quad A_{1}=\frac{1}{1+2 K+K^{2}}
$$




$$
A_{2}=\frac{2 K}{1+2 K+2 K^{2}} \quad A_{3}=\frac{K^{2}}{1+2 K+2 K^{2}}
$$

where

$$
K=e^{-\zeta \pi / \sqrt{1-\zeta^{2}}}
$$

To handle higher vibration modes, an impulse sequence for each vibration mode can be designed independently. Then the impulse sequence can be convoluted together to form a sequence of impulses that attenuate vibration at higher modes.

\subsection{Adaptive Command Shaping Using GA}

In this paper, an evolutionary method is introduced to adapt a command shaper used for vibration control of the TRMS. In the adaptation process, GA is used as a direct approach that optimizes the amplitudes of the occurring impulses and their corresponding time-locations. The general formula that represents the adapted command shaper is described as follows (Sungsoo, 1999):

$$
C(z, n)=\hat{A}_{1}(n)+\hat{A}_{2}(n) z^{-\Delta}+\cdots+\hat{A}_{m}(n) z^{-m \Delta}
$$

where $\sum_{i=1}^{m} \hat{A}_{i}=1$ and $m=1,2, \cdots$

As shown in Figure 15, the adaptation error $e(n)$ at a time $n$ is the difference between the desired response $d(n)$ and the actual system response $y(n)$. For zero vibration (ZV) with a 3-impulse command shaper, it is expected that the responses of the system due to the impulses would cancel each other out after the time $2 \Delta$. As a result the vibration would be zero after the time $2 \Delta$. Accordingly, the system response considered here after time $2 \Delta$ as the error and used to formulate the objective function for the GA optimization. The sum of squared error (SSE) is used as an objective function for adaptation of the proposed command shaper for zero vibration. This is given as: $S S E=\sum_{n=m \Delta}^{N}(e(n))^{2}$. Initially, the fixed time intervals as described in Eq.
(11)

are used and amplitudes of the impulses of the command shaper are optimized. The parameters of the GA optimization process are presented in Table 2: 
TABLE 2

GA optimization parameters

\begin{tabular}{|c|c|}
\hline GA Parameters & Values \\
\hline Initial population & 20 \\
\hline Total Generation & 50 \\
\hline Crossover rate & 0.9 \\
\hline Mutation rate & 0.001 \\
\hline Precision & 18 bits \\
\hline Objective function & Sum of squared error (SSE) \\
\hline
\end{tabular}

The program begins with a randomly generated initial population of 20 sets of values, which represent the amplitudes of impulses. Each set is assigned with the command shaper, convolved with the input bang-bang signal, and applied to the system. Then the error is calculated and the objective function is formed for this set. This procedure is repeated for each set and the corresponding objective functions recorded. Then the GA operators, namely selection, crossover, and mutation are employed to form the new population for the next generation. From the basic characteristics of GAs, it was observed that the objective function (SSE), which is the vibration in this work, reduces significantly after each generation. At the end of 50 generations, a substantial reduction in vibration is obtained.

In the next part of the work, fixed amplitudes from Eq. (11) of the impulses are used and the time intervals (locations of the impulses) are optimized. Here the time intervals are restricted to up to 20 sample instants to get reduced vibration with a reasonable delay in the system response. Higher sample intervals result huge delay in the system response. In the last part of the work both the amplitudes and timelocations of the impulses are optimized.

\subsection{Results and Discussion}

This section presents the performance of the proposed control schemes, implemented for vibration control in the vertical movement of the TRMS. To investigate the amount of vibration reduction achieved by the employed control 
schemes, the corresponding results are presented in both time and frequency domains.

Initially, three input shapers comprising 2-impulses, 3-impulses and 4-impulses were designed and implemented for vibration control in the vertical movement of the TRMS. A comparative study was conducted for the purpose of selecting the best input shaper that has a faster response and less vibration. The three input shapers, shown in Figure 17, were designed based on the vibration frequency and damping ratio of the main rotor system.

A damping ratio of 0.04146 was analytically obtained from the extracted transfer function of the system, given as (Aldebrez et al. 2004):

$$
\frac{y(s)}{u(s)}=\frac{-0.08927 s^{3}+2.249 s^{2}-45.57 s+595.1}{s^{4}+3.469 s^{3}+519.6 s^{2}+35.95 s+2189}
$$

where $u(s)$ represents main rotor input (volt) and $y(s)$ represents pitch angle (radians). This corresponds to the main resonance frequency $(0.3516 \mathrm{~Hz})$. This transfer function will be utilized in this work for simulating the system.

It can be seen from Figure 17 that the 3-impulse input shaper has achieved a better performance than the other two in terms of speed of response and vibration reduction. Thus, the 3-impulse input shaper is used for pre-processing the input signal applied to the system in an open-loop configuration. A single-switch bangbang input signal, referred to as unshaped input, used in this work is shown in Figure 18. 


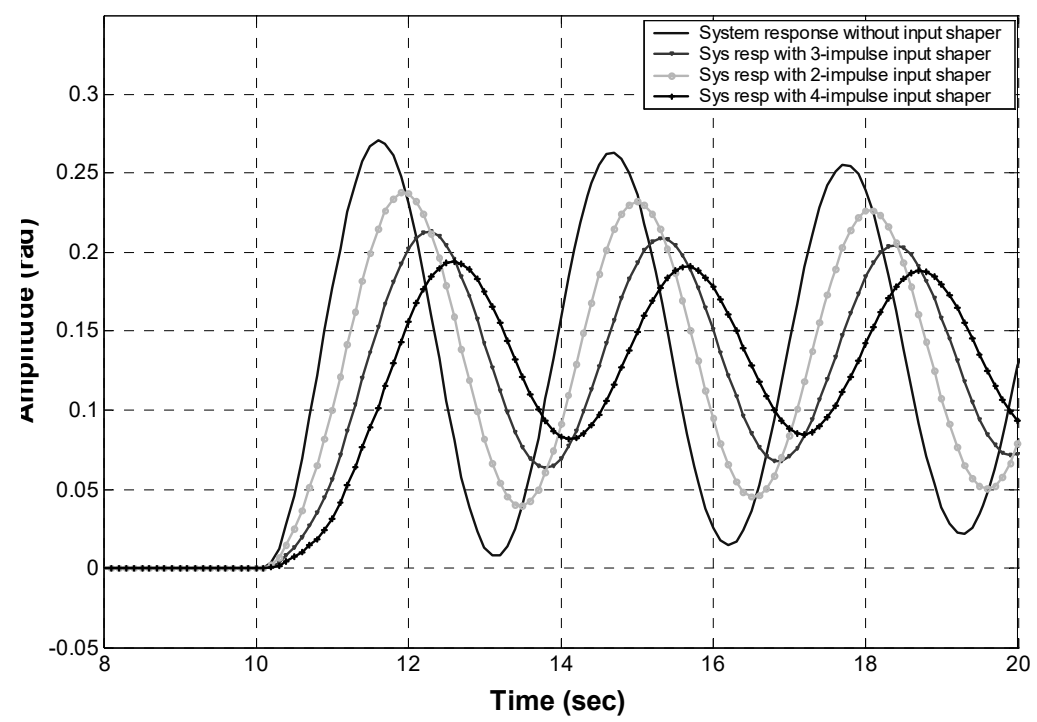

Fig. 17: Performance with 2, 3, and 4-impulse input shapers - system response without input shaper (upper curve);

- ${ }^{-}$- system response with 2 input shapers (second curve);

- - system response with 3 input shapers (third curve);

- •- system response with 4 input shapers (bottom curve).

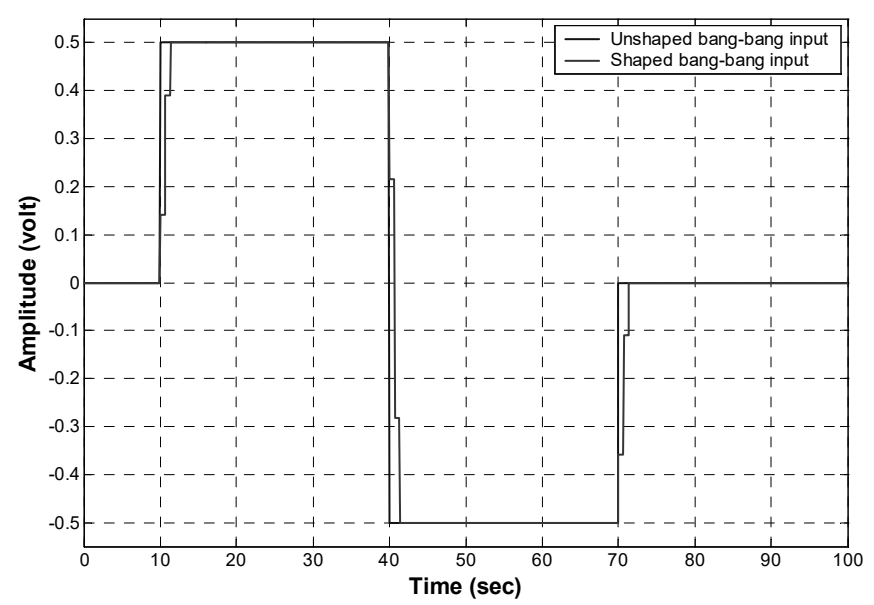

Fig. 18: Shaped and unshaped bang-bang input signals. Left line - unshaped bang bang input; right line - shaped bang bang input 
TABLE 3

Amplitudes and time-locations of the developed input shapers

\begin{tabular}{lccccccc} 
& \multicolumn{3}{c}{ Amplitudes (volts) } & \multicolumn{3}{c}{ Time locations (sec) } \\
Command Shaping Techniques & $a_{1}$ & $a_{2}$ & $a_{3}$ & $t_{1}$ & $t_{2}$ & $t a_{3}$ \\
& 0.151 & 0.398 & 0.349 & 0 & 0.7 & 1.4 \\
Standard 3-impulse IS & 0.250 & 0.50 & 0.249 & 0 & 0.7 & 1.4 \\
Adaptive 3-impulse IS (amplitudes) & 0.151 & 0.398 & 0.349 & 0 & 1.5 & 3.0 \\
Adaptive 3-impulse IS (time locations) & 0.184 & 0.490 & 0.249 & 0 & 1.4 & 2.8
\end{tabular}

TABLE 4

Rise time of the employed command shaping techniques

\begin{tabular}{|l|c|}
\hline Employed Techniques & $\begin{array}{l}\text { Rise } \\
\text { time } \\
\text { (sec) }\end{array}$ \\
\hline System response without IS & 0.775 \\
\hline Standard 3-impulse IS & 1.355 \\
\hline Adaptive 3-impulse IS (amplitudes) & 1.42 \\
\hline Adaptive 3-impulse IS (time) & 2.46 \\
\hline $\begin{array}{l}\text { Adaptive 3-impulse IS (amp \& } \\
\text { time) }\end{array}$ & 2.53 \\
\hline
\end{tabular}

The magnitude and time location of the impulses of the 3-impulse input shaper were obtained by solving Eq. (12). Table 3 shows the amplitudes of the three impulses and their corresponding time locations. For discrete implementation of the input-shaper, locations of the impulses were selected at the nearest sample timestep.

The corresponding system responses to the applied shaped and unshaped input signals are shown in Figure 19. With the three-impulse sequence, the oscillations in the system response were found significantly reduced. These can be observed by comparing the system response to the unshaped input. Figure 19(a) shows the system response for the duration of experiment (100 sec), while Figure 19(b) represents the operation from 10 to 40 seconds. It can be seen from this figure that there is a significant amount of vibration reduction achieved with the employed 
controllers in comparison to the system without command shaping.

To investigate time domain response of the system with the employed techniques, rise time was recorded, see Table 4. The system response without

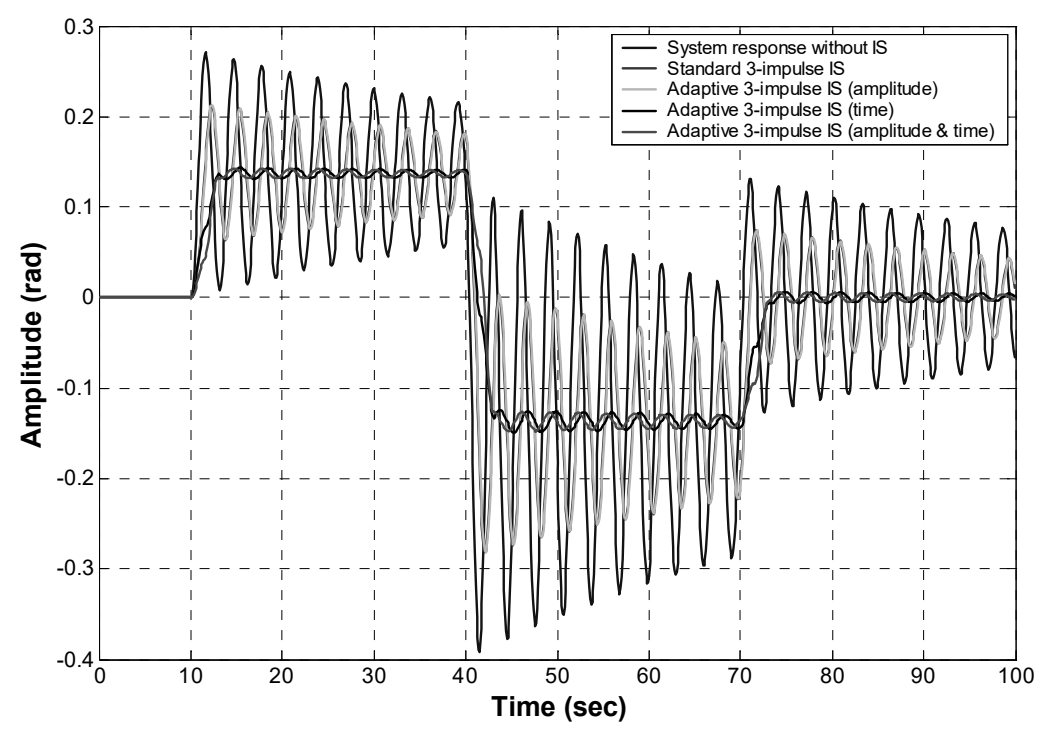

(a) Time domain response over the complete cycle 


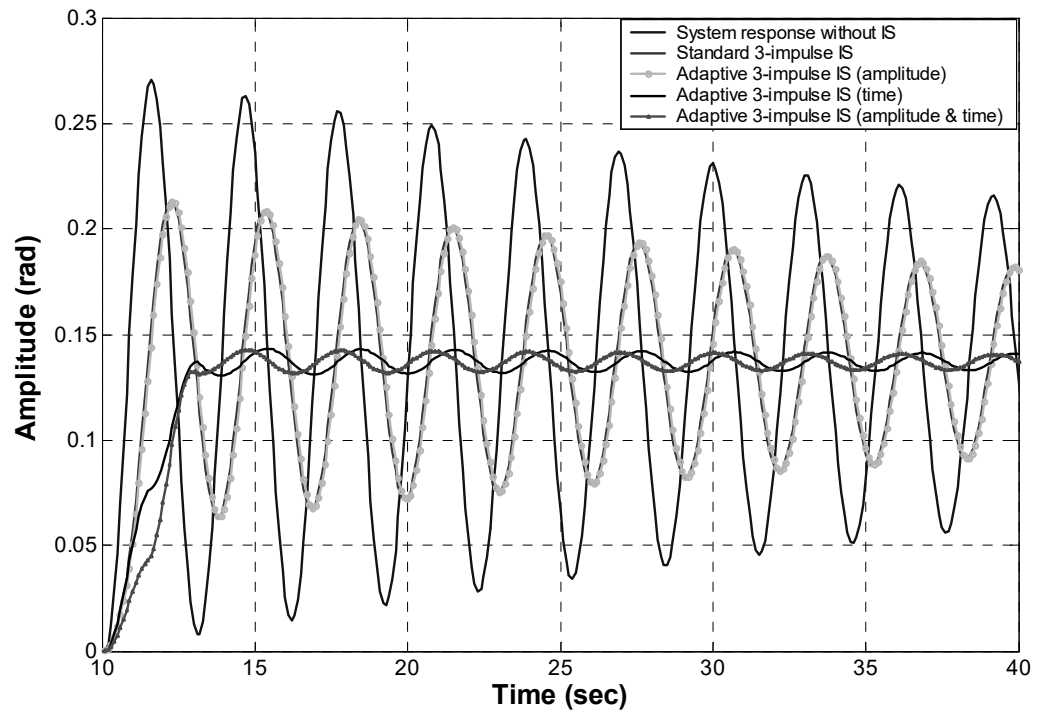

(b) Time domain response from 10 to 40 secs

Fig. 19: Performance of the employed 3-impulse input shapers command shaping has recorded the shortest rise time of $0.7775 \mathrm{sec}$, followed by that with the standard input shaper with a rise time of $1.355 \mathrm{sec}$. However, the adaptive input shapers have recorded slow system response with rise times of $1.42 \mathrm{sec}, 2.46$ sec and $2.53 \mathrm{sec}$ for adaptive amplitude, adaptive time-location and adaptive amplitude and time-location, respectively.

Generally, the obtained results show that the introduction of command shaping resulted in a delay in the system response. This delay is equivalent to the convoluted impulses during the shaping process. This can be attributed as a main drawback of the command shaping algorithms in general. To obtain a faster system response, a feedback control method would be augmented with the command shaping algorithm.

It can also be noticed from the power spectral density (PSD) plot shown in Figure 20 and vibration reduction in Table 5 that vibration of the system was dramatically reduced. As shown in Figure 20(a), the system has a single dominant mode at $0.3516 \mathrm{~Hz}$. Since, this work focuses on the vibration control of the system, the spectral attenuation at the dominant mode region is recorded in Table 5 and plotted in Figure 20(b).

A comparative performance of the system with the standard 3-impulse input 
shaper, adaptive amplitude 3-impulse input shaper, adaptive time-location 3-impulse input shaper and adaptive amplitude-time 3-impulse input shaper is presented in Table 5. It is evident from the results that the standard and the three adaptive input shapers have performed well in reducing the system vibrations. Among the employed controllers, the best result in terms of vibration reduction was achieved with the adaptive amplitude-time input shaper $(65.41 \mathrm{~dB})$, followed by the adaptive time input

TABLE 5

Amount of vibration reduction with the employed 3-impulse input shapers

\begin{tabular}{|l|c|}
\hline $\begin{array}{l}\text { Employed Command Shaping } \\
\text { Techniques }\end{array}$ & Vibration amount (dB) \\
\hline Standard 3-impulse IS & 11.11 \\
\hline Adaptive 3-impulse IS (amplitudes) & 11.20 \\
\hline Adaptive 3-impulse IS (time) & 57.95 \\
\hline Adaptive 3-impulse IS (amp \& time) & 65.41 \\
\hline
\end{tabular}

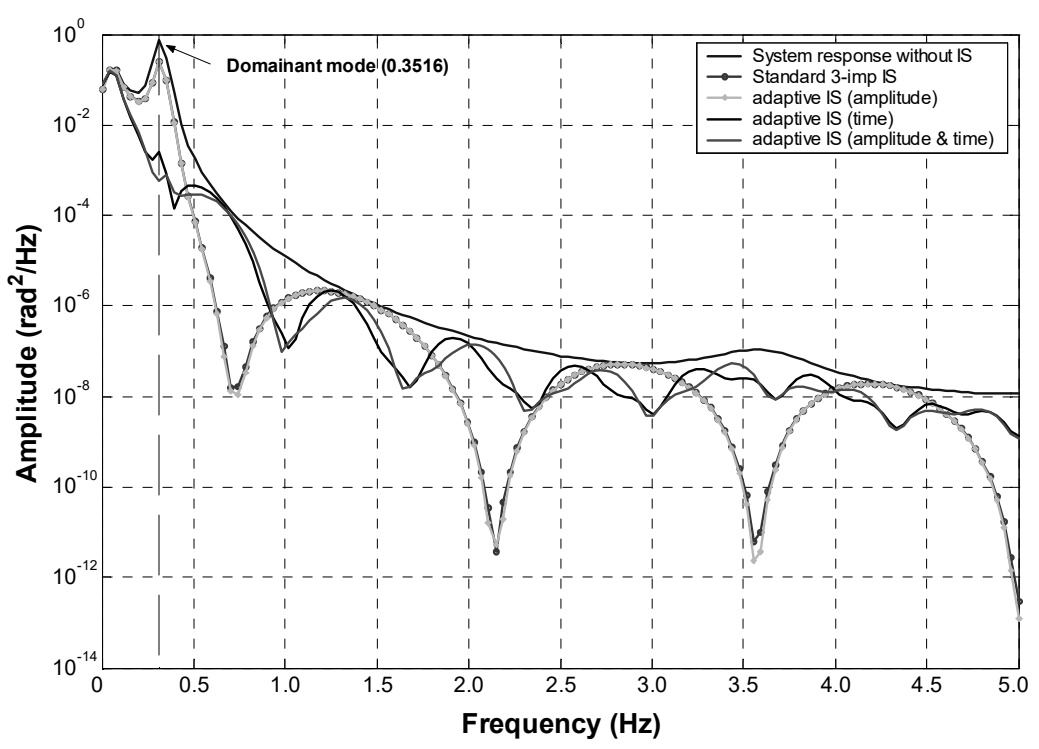

(a) PDS for the whole duration 


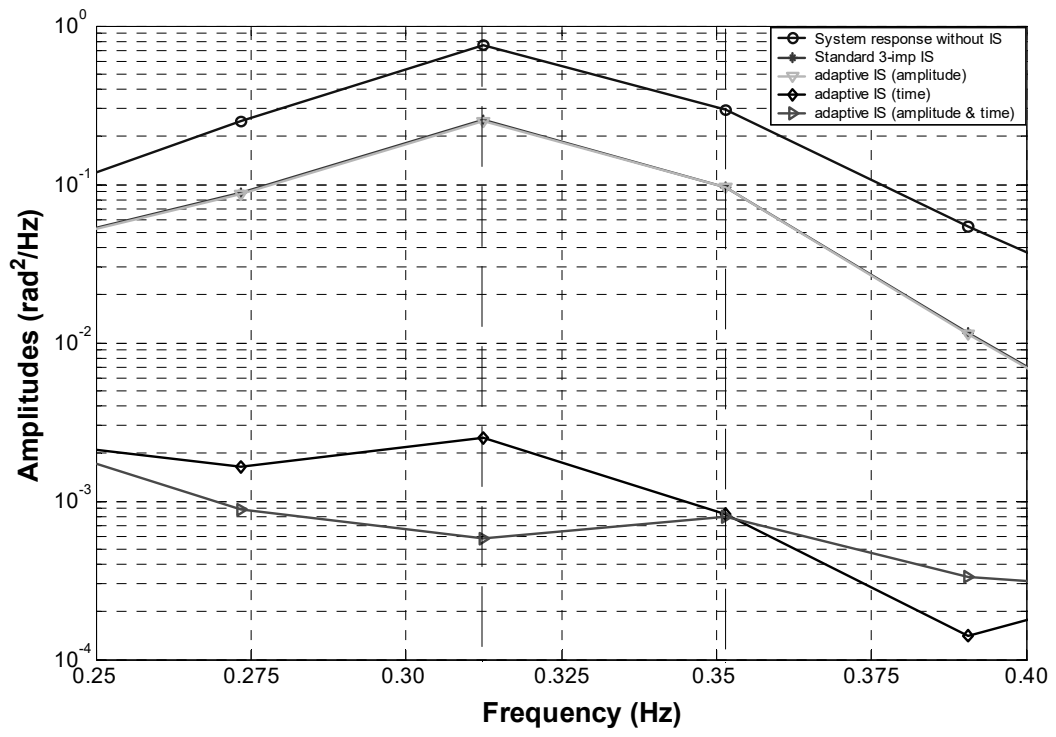

(b) PSD for the dominant region

Fig. 20: Power spectral density

shaper with $57.95 \mathrm{~dB}$, and then the adaptive amplitude input shaper with $11.20 \mathrm{~dB}$, and finally the standard 3-impulse input shaper with $11.11 \mathrm{~dB}$ reduction.

Figure 21 shows the GA objective function profile, sum of squared error (SSE), of the three adaptive input shapers. The change in the objective function for the adaptive amplitude input shaper is not significant in comparison to the other two adaptive input shapers. Table 6 shows the final values of the SSE at the end of 50 generations. 


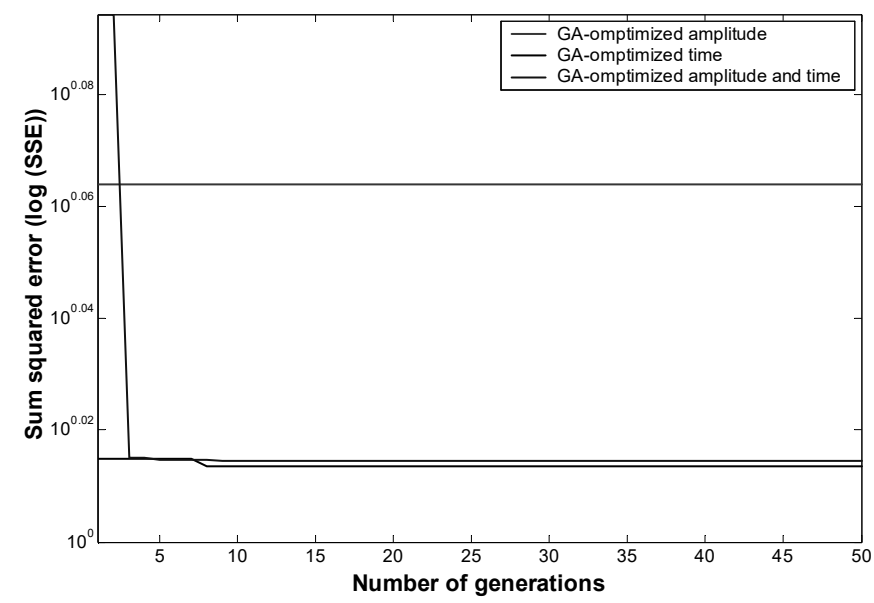

Fig. 21: GA objective function (SSE).

Legend: - - - GA optimized amplitude; - - - GA optimized time; — GA optimized amplitude and time;

\section{TABLE 6}

GA objective function

\begin{tabular}{|l|c|}
\hline Adaptive Input Shapers & Values of SSE after 50 generations \\
\hline Adaptive 3-impulse IS (amplitudes) & 14.4116 \\
\hline Adaptive 3-impulse IS (time) & 10.7588 \\
\hline Adaptive 3-impulse IS (amp \& time) & 10.8138 \\
\hline
\end{tabular}

\section{CONCLUSION}

Genetic algorithm (GA) is inspired by the mechanism of natural selection that emulates the laws of evolution and genetics to yield optimal solution to complex problems. This paper has presented an investigation into utilization of GA in the field of control engineering, specifically in tracking control and vibration suppression. The potential of GA has been assessed in three case studies.

Case 1 presented the development of hybrid learning control schemes with GA for input tracking and vibration suppression of a flexible manipulator. The control 
scheme has been implemented and tested within simulation and experimental environments and its performance has been evaluated in terms of input tracking capability and vibration suppression at the resonance modes of the manipulator. An acceptable input tracking control and vibration suppression have been achieved with the proposed control strategy.

In Case 2, the design and implementation of an adaptive active control mechanism using GA have been presented and verified through simulated exercises in a flexible fixed-free beam system. The performance of the control system in vibration reduction with different types of excitation has been assessed. It has been demonstrated that on average, a significant amount of vibration reduction over the full range of frequencies of the input signal has been achieved.

In Case 3, two types of command shaping methods, namely, standard input shaper and GA-based adaptive command shaper have been developed and investigated for vibration control in the vertical movement of a TRMS. Significant improvement in the reduction of system vibration has been achieved with the employed techniques as compared to the system with unshaped bang-bang input. Among the four employed command shapers, the best performance has been achieved with the adaptive amplitude and time 3-impulse input shaper.

We have shown that when locations of the impulses are fixed at the theoretical value there is no significant reduction in vibration compared to theoretical command shaping even though the amplitudes are varied. Speed of response depends on the locations of impulses, not on the amplitudes of the impulses. When the amplitudes of impulses are fixed at theoretical values but the locations are varied, a significant amount of reduction in vibration can be achieved at the cost of long delay. It is noted from the obtained results that there is a delay in the system response as compared with that using unshaped input. This is equivalent to the length of the impulse. This can be tackled by augmenting command shaping with a feedback control method.

\section{REFERENCES}

Aldebrez F.M., Alam M.S. and Tokhi M.O. (2004). Genetic modelling and vibration control of a twin rotor system, UKACC International Conference on control, Bath, UK. 
Aldebrez F.M., Tokhi M.O., Mohamed Z. and Ahmad M.S. (2003). Vibration control of pitch movement using command shaping techniques, Proc. IEEE Emerging Technologies and Factory Automation Conference (ETFA'03), Lisbon, Portugal, 1, 447-452.

Arimoto S., Kawamura S., and Miyazaki F. (1984). Bettering operation of robots by learning, Journal of Robotic Systems, 1(2), 123-140.

Bodson M. (1998). An adaptive algorithm for the tuning of two input shaping methods, Automatica, 34(6), 771-776.

Chen, B.S. and Cheng, Y.M. (1998). A structure-specified H-infinite optimal control design for practical applications: a genetic approach, The IEEE Transcation on Control Systems Technology, 6(6), 707-718.

Dakev, N.V., Whidborne, J.F., Chipperfield, A.J. and Fleming, P.J. (1997). Evolutionary $\mathrm{H}$-infinity design of an electromagnetic suspension control system for a maglev vehicle, Proceedings of the Institution of Mechanical Engineers Part I, 211, 345-355.

Goldberg, D.E. (1989). Genetic Algorithm in Search, Optimization and Machine Learning, Addison-Wesley, Reading, MA.

Holland J.H. (1975). Adaptation in natural and artificial systems, University of Michigan Press, Ann Arbor MI, University Michigan Press.

Hossain, M.A. (1996). Digital signal processing and parallel processing for realtime adaptive active vibration control, $\mathrm{PhD}$ Thesis, Department of Automatic Control and Systems Engineering, University of Sheffield, UK.

Hossain, M.A. and Tokhi, M.O. (1997). Evolutionary adaptive active vibration control, Proc. of IMechE-I: Journal of Systems and Control Engineering, 211(I3), 183-193.

Hossain, M.A., Tokhi, M.O., Chipperfield, A.J., Baxter, M.J., Fonseca, C.M. and Dakev, N.V. (1995). Adaptive active vibration control using genetic algorithms, First International Conference on Genetic Algorithms in Engineering Systems: Innovations and Applications (GALESIA), 12-14 Sept. 1995, 175-180.

Ichikawa, Y., and Sawa, T. (1992). Neural Network Application for Direct Feedback Controllers, IEEE Transactions on Neural Networks, 3(2), 224-231.

Itoh, K., Iwasaki, M. and Matsui, N. (2004). Optimal design of robust vibration suppression controller using genetic algorithms, Engineering systems with Intelligence concepts, Tools and Applications, 431-438. Kourmoulis, P.K. (1990). Parallel processing in the simulation and control of flexible beam structure system, PhD. Thesis, Department of Automatic Control and Systems Engineering, University of Sheffield, UK.

Kristinsson, K. and Dumont, G. (1992). System identification and control using genetic algorithms, The IEEE Transactions on Systems, Man and Cybernatics, 22(5), 1033-1046.

Leung, F.H.F., Lam, H.K., Ling, S.H. and Tam, P.K.S. (2003). Tuning of the structure and parameters of a neural network using an improved genetic algorithm, IEEE Transactions on Neural Networks, 14(1), 79-88. 
F.M. Aldebrez, et al.

Linkens, D.A. and Nyongesa, H.O. (1996). Learning systems in intelligent control: an apprisal of fuzzy, neural and genetic control applications, IEE ProceedingsControl Theory and Applications, 143(4), 367-386.

Linkens, D.A., and Nyongesa, H.O. (1995). Genetic algorithms for fuzzy control Part 1: Offline system development and application and Part 2: Online system development and application. IEE Proceedings - Control Theory and Applications, 142(3), 161-176 and 177-185.

Luh, G.C. and Wu, C.Y. (1999). Non-linear system identification using genetic algorithms, Proceedings of the Institution of Mechanical Engineers Part I, 213, 105- 118 .

Mei, T.X. and Goodall, R.M. (2000). LQG and GA solutions for active steering of railway vehicles, IEE Proceedings - Control Theory and Applications, 147(1), 111-117.

Mohamed Z. and Tokhi M.O. (2002). Vibration control of a single-link flexible manipulator using command shaping techniques, Proc. Inst. Mech. Eng., Part I, $J$ Systems and Control Engineering, 216, 191-210.

Mohamed, Z. and Tokhi, M.O. (2002). A symbolic manipulation approach for modelling and performance analysis of flexible manipulator systems, International Journal of Acoustics and Vibration, 7(1), 27-37.

Mohamed, Z. and Tokhi, M.O. (2004). Command shaping techniques for vibration control of a flexible robot manipulator, Mechatronics, 14, 69-90.

Oliveira, P., Sequeira, J. and Sentieiro, J. (1991). Selection of Controller Parameters using Genetic Algorithms, Engineering Systems with Intelligence. Concepts, Tools, and Applications, 431-438.

Onnen, C., Babuška, R., Kaymak, U., Sousa, J.M., Verbruggen, H.B. and Isermann, R. (1997). Genetic algorithms for optimization in predictive control. Control Engineering Practice, 5(10), 1363-1372.

Patterson, W. (1996). Artificial Neural Networks: Theory and Applications. Prentice Hall, Singapore.

Rana, A.S. and Zalzala, A.M.S. (1997). Collision-free motion planning of multiarm robots using evolutionary algorithms, Proceedings of the Institution of Mechanical Engineers Part I, 211, 373-384.

Singer N.C. and Seering W.P. (1990). Preshaping command inputs to reduce system vibration, Trans. ASME, J. Dynamic Systems, Measurement and Control, 112(1), 76-82.

Singhose W.E., Singer N.C. and Seering W.P. (1995). Comparison of command shaping methods for reducing residual vibration, Proc. European Control Conference, Rome, 1126-1131.

Sungsoo R. (1999). Adaptive command shaping using adaptive filter approach in time domain, Proc. American Control Conference, San Diego, California, 8185.

Tokhi, M.O. and Azad, A.K.M. (1996). Control of flexible manipulator systems. Proceedings of IMechE - I: Journal of Systems and Control Engineering, 210(I2), 113-130. 
Tokhi, M.O. and Azad, A.K.M. (1997). Design and development of an experimental flexible manipulator system, Robotica, 15(3), 283-292.

Tokhi, M.O. and Veres, S.M. (2002). Active sound and vibration control - Theory and applications, Institution of Electrical Engineers, London, 2002.

Tokhi, M.O., Azad, A.K.M. and Poerwanto, H. (1999). SCEFMAS: An environment for dynamic characterisation and control of flexible robot manipulators. International Journal of Engineering Education, 15(3), 213-226.

Tokhi, M.O., Mohamed, Z. and Azad, A.K.M. (1997). Finite difference and finite element approaches to dynamic modelling of a flexible manipulator, Proceedings of IMechE-I: Journal of Systems and Control Engineering, 211(I2), 145-156.

Varsek, A., Urbanèiè, T. and Fillipiè, B. (1993). Genetic Algorithms in Controller Design and Tuning. IEEE Transactions on Systems, Man, and Cybernetics, 23(5), 1330-1339.

Whidborne, J.F., Postlethwaite, I. and Gu D. -W. (1994). Robust controller design using $\mathrm{H}$ loop-shaping and the method of inequalities, IEEE Trans. On Contr. Syst. Technology, 2(4), 455-461. 